\title{
Coulisses
}

Revue de théâtre

19 | Hiver 1999

Varia

\section{La farce au Moyen Age et chez Molière et ses prolongements modernes}

\section{Thierry Miguet}

\section{(2) OpenEdition}

1 Journals

Édition électronique

URL : https://journals.openedition.org/coulisses/5537

DOI : $10.4000 /$ coulisses. 5537

ISSN : 2546-9460

\section{Éditeur}

Presses universitaires de Franche-Comté

\section{Édition imprimée}

Date de publication : 1 janvier 1999

Pagination : 31-34

ISBN : 2-913322-09-3

ISSN : $1150-594 X$

\section{Référence électronique}

Thierry Miguet, "La farce au Moyen Age et chez Molière et ses prolongements modernes ", Coulisses [En ligne], 19| Hiver 1999, mis en ligne le 18 octobre 2019, consulté le 04 janvier 2022. URL : http:// journals.openedition.org/coulisses/5537 ; DOI : https://doi.org/10.4000/coulisses.5537

Ce document a été généré automatiquement le 4 janvier 2022.

Coulisses 


\title{
La farce au Moyen Age et chez Molière et ses prolongements modernes
}

\author{
Thierry Miguet
}

\section{Introduction. Une salutaire détente ; récréation recréation}

1 Le Moyen Age n'a pas inventé la farce, car le drame satyrique de l'Antiquité grecque la précède ; mais il l'a retrouvée, et son origine, comme celle du théâtre d'Athènes tout entier, est religieuse. Comme la représentation scénique des souffrances de Dionysos est un moment égayée par une pièce, où dominent les hilarantes pirouettes et saillies des satyres, de même le jeu de la passion du Christ est momentanément interrompu par le rythme endiablé d'un intermède bouffon; pour désigner cette interruption, qui est une détente salubre au milieu de la tension du «mystère », les gens d'Eglise ont choisi une image culinaire, et l'ont appelée "farce ", d'après le participe passé "farsa ", forme populaire du latin classique « farta », remontant au verbe « farcire », " farcir ».

2 Dans un passage consacré à la farce médiévale, extrait de son étude intitulée Le théâtre, Marie-Claude Hubert a ces remarques pertinentes, qui éclairent et confirment notre propos :

Cent cinquante farces, environ, nous sont parvenues, dont la composition s'échelonne entre 1440 et 1560 . Intégrés à un spectacle sérieux, une moralité ou un mystère, ces divertissements populaires, destinés à faire rire, le «farcissaient » d'éléments comiques. C'est ce qui fait dire à Molière, dans la préface du Tartuffe, que la « comédie, chez les Anciens, a pris son origine dans la religion et faisait partie de leurs mystères ${ }^{1}{ }^{1}$

3 De ce lien existant entre la plus sainte sagesse et la plus franche récréation, nous détenons une preuve d'une éminente dignité. Elle nous vient du premier et du plus 
illustre des Pères du désert, de saint Antoine en personne. Voici le récit qui lui donne la parole :

Il y avait dans le désert un chasseur de bêtes sauvages qui vit abba Antoine se récréant avec des frères. Il s'en scandalisa. Voulant le convaincre qu'il fallait de temps en temps condescendre aux frères, le vieillard lui dit : « Mets une flèche à ton arc, et bande-le. » Il fit ainsi. Le vieillard reprit: «Bande-le un peu plus », et le chasseur le fit. Le vieillard lui dit encore: "Continue à le bander.» Le chasseur répondit : « Si je bande mon arc au-delà de la mesure, je vais le casser.» Le vieillard lui dit alors: «Il en va de même dans l'œuvre du Seigneur; si nous tendons les frères outre mesure, ils seront bientôt brisés. Il faut donc de temps en temps condescendre à leurs besoins. » Entendant ces paroles, le chasseur fut pénétré de componction. Grandement édifié par le vieillard, il partit. Quant aux frères, ils retournèrent chez eux fortifiés. $»^{2}$

Farces (création collective du TUFC, 1998)

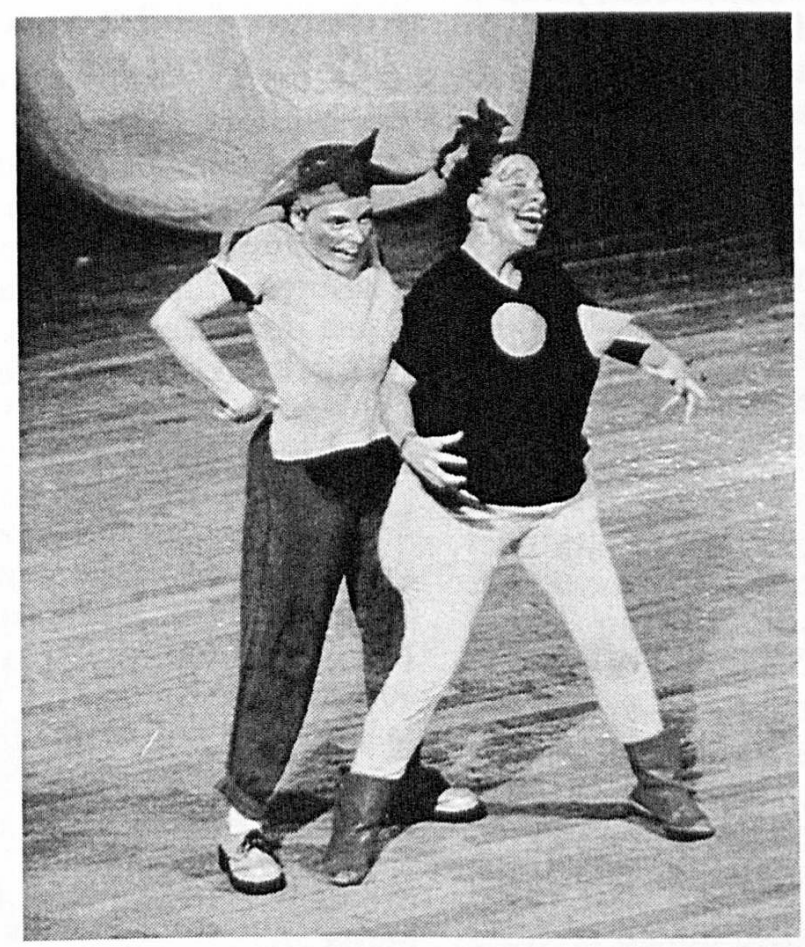

Photo Georges Pannetton

4 Après une telle lecture, on peut dire, en parodiant La Fontaine : la farce fait passer le tragique avec elle; le plaisant débridé qui est en elle nous dispose à l'accueil du grave qui l'entoure; on constate, pour peu qu'on y songe, que, par son utilité humaine et compréhensive, cet éclat de rire porté sur les planches sauvegarde (l'arc n'est pas brisé), renouvelle (l'attention reposée est restaurée), édifie (engendrer la bonne humeur est générosité), et fortifie (qui a ri a vaincu le destin) le spectateur de la désopilante saynète. 


\section{Le ressort de la « machine à rire »(Rey Flaud) : la gambade}

Deux paroles de Montaigne suggèrent ce que l'on pourrait appeler l'âme de la farce :

- «Cette farcissure est un peu hors de mon thème ; je m'égare, mais plutôt par licence que par mégarde ».

- «J'aime l'allure poétique, à sauts et à gambades.$^{3}$

6 Certes, le mouvement vif est essentiel à ce genre littéraire : cabriole ou culbute, esquive ou fuite, arrivée ou départ en coup de vent, pas de danse ou pas de course, précipitation à répétition, bonds d'allégresse, explosion de pétulance amoureuse, la farce met sous nos yeux l'éclosion d'une exultation du corps et du cœur. Exulter voulant dire " sauter ", la farce est une « sauterie " qui célèbre une victoire : la victoire d'un malin sur un benêt, d'un futé sur une dupe.

7 Cependant toutes les variantes sont possibles. Ainsi dans la farce médiévale du Cuvier, ce n'est pas le vainqueur, Jacquinot, qui saute de joie, alors qu'il est en train de prendre une revanche sur son épouse, dragon domestique; c'est elle qui fait tous les soubresauts imaginables pour sortir de la cuve à linge pleine d'eau, où elle vient de tomber; elle a beau demander de l'aide à son mari, celui-ci ne lui porte pas secours, parce qu'une telle intervention ne figure pas sur la longue liste des tâches ménagères, que lui ont dictées sa femme et sa belle-mère, sur son « rollet », selon le mot sans cesse repris du héros de l'aventure ; l'échange suivant suffit à valoriser le terme :

- LA FEMME. Jacquinot, sauvez votre femme ;

Tirez-la hors de ce baquet.

- JACQUINOT. Cela n'est point en mon rollet.

Maître Pathelin, dans la farce qui porte son nom, joue si bien le délire, en présence du drapier qui vient lui réclamer son argent, que celui-ci, terrifié, prend la fuite sans avoir été payé. Là aussi, le repli brusque et imprévu est celui de la victime, et non celui du meneur de jeu.

Mais dans la farce du Poullier, c'est le meunier trompeur qui déclenche toute l'intrigue mouvementée, jusqu'à obliger le spectateur à donner au mot «sauter » son sens le plus grivois. Voici en effet l'analyse de ce « chef-d'œuvre » par Pierre Voltz :

Deux personnages rusés et sans scrupules, un meunier et sa femme, y bernent deux gentilshommes dans un sujet d'une audace digne des contes italiens les plus osés. Pour se venger de ce qu'ils courtisent sa femme, le meunier leur fait donner par elle rendez-vous au moulin. Le premier, délogé par le second, se réfugie dans un poulailler; le second, dérangé par le mari, y rejoint le premier et ils assistent, impuissants, à la vengeance du [meunier], qui empoche leur argent, mange le repas qu'ils avaient apporté et invite les deux «demoiselles", leurs femmes, qui se montrent complaisantes au-delà de toute espérance, sous les yeux de leurs maris. ${ }^{4}$

10 La farce est ici portée à son comble, puisque les deux aristocrates bernés voient, depuis la maison des poules, leurs conjointes, comme des gourgandines, se "farcir» le meunier, qui les « saute».

11 Molière connaît la chanson : nul personnage de théâtre ne saute plus souvent, ni plus adroitement, par la fenêtre que le Sganarelle du Médecin volant; d'où la remarque de Gros-René, éberlué : «Ah ! ma foi, voilà qui est drôle ! comme diable on saute ici par les fenêtres!» 

maître de façon savoureuse: "Voilà le coquin qui faisait le médecin, et qui vous trompe. Cependant qu'il vous trompe et joue la farce chez vous, Valère et votre fille sont ensemble, qui s'en vont à tous les diables. »

érobee apparaît dans ce passage le génie de Molière : il place le mot « farce » dans sa propre farce, « en abîme ", et tend à lui donner déjà le sens moderne d'« attrape », de "petite tromperie par plaisanterie», ce sens enjoué que Flaubert adopte sans le dire dans son Dictionnaire des idées reçues: «Farce. Il faut en faire lorsqu'on est en partie de campagne avec des dames. »

Dans La Jalousie du Barbouillé, l'autre farce des débuts de Molière, c'est un comportement de fuite, habile et preste, qui est envisagé et réalisé par Angélique, puis plaisamment commenté par son mari, le Barbouillé. Celui-ci refusait d'ouvrir la porte de leur demeure à son épouse, revenue trop tard du bal ; elle feint de se suicider, ce qui oblige le mari à venir vérifier la chose, avec la " chandelle ", car la nuit est complète. Le moment est venu de donner la parole à nos héros :

- ANGÉLIQUE. II faut que je t'attrape. Si je peux entrer dans la maison subtilement, cependant que tu me chercheras, chacun aura bien son tour.

- LE BARBouilLé. Hé bien! ne savais-je pas bien qu'elle n'était pas si sotte? Elle est morte, et si (= pourtant) elle court comme le cheval de Pacolet (= nain qui, par art magique, avait «fait un cheval en bois qui allait par les airs plus vite qu'un oiseau »). Ma foi, elle m'avait fait peur tout de bon. Elle a bien fait de gagner au pied ; car si je l'eusse trouvée en vie, après m'avoir fait cette frayeur-là, je lui aurais apostrophé cinq ou six clystères de coups de pied dans le cul, pour lui apprendre à faire la bête.

Mais voici notre péroreur à son tour devant sa porte close, et trouvant sa femme aussi intraitable envers lui, qu'il l'a été envers elle.

Il est facile de prouver, par quelques touches opportunes, que ces bonds et ces courses se trouvent dans le drame satyrique. Le Silène du Cyclope d'Euripide est prêt à faire un plongeon vertigineux pour avoir le droit de s'enivrer avec la liqueur de Bacchus : « Oui, je raffolerais de vider une coupe, une seule, en échange du bétail de tous les Cyclopes, et de sauter dans l'onde amère, pour un moment d'ivresse où les sourcils se détendent. » (vers 164-7)

Un peu plus loin, le coryphée des satyres souhaite que Polyphème ne l'avale pas avec le lait des jarres; voici la réponse du cyclope : «Nul danger! À me sauter au milieu du ventre, vous me tueriez par vos gambades. » (vers 220-1)

18 Le drame satyrique de Sophocle, Les limiers, met en scène Silène et ses enfants, les satyres, à la poursuite d'un mystérieux voleur, qui a dérobé les génisses d'Apollon; l'auteur du larcin se révèle être le fils de Zeus et de Maïa, le dieu Hermès, qui vient par ailleurs d'inventer la première lyre. Dès le prologue, Apollon s'écrie : «Cette poursuite, cette chasse me passionne. » (vers 15)

Cependant de la caverne du divin prédateur, vers laquelle les traces de la piste mènent «les limiers ", sortent les sons étranges d'un instrument inconnu ; Silène alors prend peur et s'enfuit, comme le drapier devant le délire de Pathelin. Plus courageux, le chœur arrive au repaire du larron musicien, et, pour que le joueur de lyre se montre, le coryphée énonce la décision qu'il a prise : «Je vais vite faire du bruit en frappant le sol, et mes souples gambades, mes ruades, le forceront bien à m'écouter, même s'il est complètement sourd. » (vers 211-4) 
ene, la nourrice du dieu, essaie d'éloigner cet importun à l'aide d'une menace, dont le sens est proverbial, vérité d'expérience, mais dont la tournure singulière établit la parenté radicale entre l'esprit du drame satyrique et l'esprit de la farce : «Ne sais-tu pas que le sot qui s'amuse aujourd'hui aux railleries et aux farces, les dieux le feront pleurer demain? » (vers 360-1)

C'est ce qu'a parfaitement compris Claudel dans son " drame satyrique en deux actes ", Protée. Écoutons la nymphe Brindosier, héroïne de cette pièce, faire à Ménélas l'éloge des satyres :

L'antilope de Syrie qui des quatre pieds sans aucun poids vient se poser sur la tête de son pâtre,

Qu'est-ce qu'elle est à côté de nos grands sauteurs ? (acte I, scène III)

Au sujet de ses satyres, qu'il a enlevés au cortège de Bacchus, Protée s'exprime ainsi :

C'est dans leur intérêt que je les conserve. Je veux leur apprendre l'hygiène et la morale.

Et puis cela m'amuse aussi de les voir sauter de roc en roc. C'est pittoresque. Il me semble que cela anime la localité ! (acte I, scène V)

Parfois c'est la didascalie qui nous renseigne sur l'ambiance bondissante ou dansante de la représentation. En témoignent les indications suivantes, qui concernent la scène VI de l'acte II : «À ce moment l'envoyé de Jupiter s'avance avec un bond gracieux. (...) Il danse avec Hélène un petit ballet dans le genre classique. »

Ce sont aussi des didascalies qui nous apprennent certains mouvements joyeux de $L a$ Savetière prodigieuse, "farce violente en deux actes et un prologue » de Federico Garcia Lorca. Ainsi cette indication de la scène XI de l'acte $\mathrm{I}$ : « Elle se lève et se met à danser avec des amoureux imaginaires. " De même, toujours dans l'acte premier, cette information de la scène XVIII, qui est une chasse au papillon avec un enfant: « SAVETIÈRE, courant aussi, de l'autre côté. » C'est encore dans une didascalie de la même pièce que l'auteur nous enseigne ce principe régissant le genre littéraire qu'il est en train d'illustrer: "La farce exige toujours le naturel.» (scène première de l'acte deuxième)

Dans Les chaises, farce tragique d'Eugène Ionesco, nous devinons la mort de Sémiramis et de son mari grâce à une didascalie: "La Vieille et le Vieux, en même temps, se jettent chacun par sa fenêtre, en criant "Vive l'Empereur". »

Dans Les caves du Vatican d'André Gide, "farce en trois actes extraite du roman par l'auteur", nous comprenons qu'Anthime Armand-Dubois vient d'être guéri miraculeusement par la Madone d'une douloureuse sciatique, grâce aux indications de cette didascalie: "Anthime relève la tête comme sortant d'un songe. Il se lève, craintivement d'abord, fait jouer ses membres, se baisse pour ramasser sa béquille tombée à terre, exécute avec elle quelques gambades ; puis la rejette. »

Dans la même pièce, par deux fois, la joie d'un escroc qui a réussi son coup se manifeste par une figure chorégraphique notée dans une didascalie : « Durant son absence (= celle de la victime, la comtesse de Saint-Prix), Protos (= le filou déguisé en chanoine) exécute un entrechat "; et un peu plus loin : «La comtesse sort un instant; durant que Protos esquisse un entrechat. » (VI' tableau)

Dans cette farce se retrouve le goût de Gide pour la mise en abîme, puisque, au cours de l'action, un personnage est amené à dire à un autre personnage : « Mon cher ami, vous 
prendrez comme vous voudrez cette aventure, moi je la tiens pour une farce qualifiée. » (XII tableau)

\section{Le lubrifiant de la « machine à rire »: le comique de mots}

Dans ce vaste domaine Molière s'amuse royalement. Ainsi le docteur de La jalousie $d u$ Barbouillé démontre avec un imperturbable brio qu'il est « une, deux, trois, quatre, cinq, six, sept, huit, neuf, et dix fois docteur. » Puis, dans une admirable tirade, animée d'un mouvement d'aller et de retour, il compose des expressions gigognes, s'emboittant donc les unes dans les autres, d'abord de la plus petite à la plus grande, puis, en sens rigoureusement inverse, de la plus grande à la plus petite. Voici ces expressions gigognes dans leur apparition ascendante :

bourse pleine de pistoles

riche boîte

étui précieux

coffret admirable

cabinet curieux

chambre magnifique

appartement agréable

château pompeux

citadelle incomparable

ville célèbre

île fertile

province opulente

monarchie florissante

tout le monde

Chacun des quatorze termes de cette progression est à la fois contenu et contenant, sauf le dernier qui n'est que contenant, mais qui est le point de départ du mouvement descendant. Et tout cet effort verbal n'est là que pour signifier au Barbouillé que le docteur se soucie aussi peu de l'argent que de son propre postérieur, qu'il exhibe en fin de tirade.

Quand Angélique, impertinente, lance au docteur: «Je me moque bien de vous et de votre doctrine, et je suis docteur quand je veux », loin de se décontenancer, le docteur prouve à la persifleuse, à l'aide de la grammaire et de la scansion latine, qu'elle ne pense qu'à ça :

Tu es docteur quand tu veux, mais je pense que tu es un plaisant docteur. Tu as la mine de suivre fort ton caprice : des parties d'oraison (= des éléments du langage), tu n'aimes que la conjonction (= la copulation); des genres, le masculin (= le mâle); des déclinaisons, le génitif (=le génital); de la syntaxe, mobile cum fixo (= d'agencement, tu n'aimes que celui de l'organe mobile avec l'organe fixe)! et enfin de la quantité (= de la métrique), tu n'aimes que le dactyle, quia constat ex una longa et duabus brevibus (= allusion à la structure du sexe masculin).

Oui, l'immense espace des jeux de mots s'ouvre à la farce, pourvu que ces jeux soient intégrés au mouvement qui l'emporte. Voici l'un de ces jeux, extrait des Chaises; c'est la vieille qui parle : « II a un air emprunté. II nous doit beaucoup d'argent. » 


\section{Conclusion}

Le meilleur de la farce médiévale a passé dans Molière, qui retrouve tous les bonds des satyres. Le bon tour qu'est la farce provoque rire et gambade ; mais celle-ci est d'autant plus alerte et entraînante et salutaire, qu'elle est huilée par le bon mot.

Farce

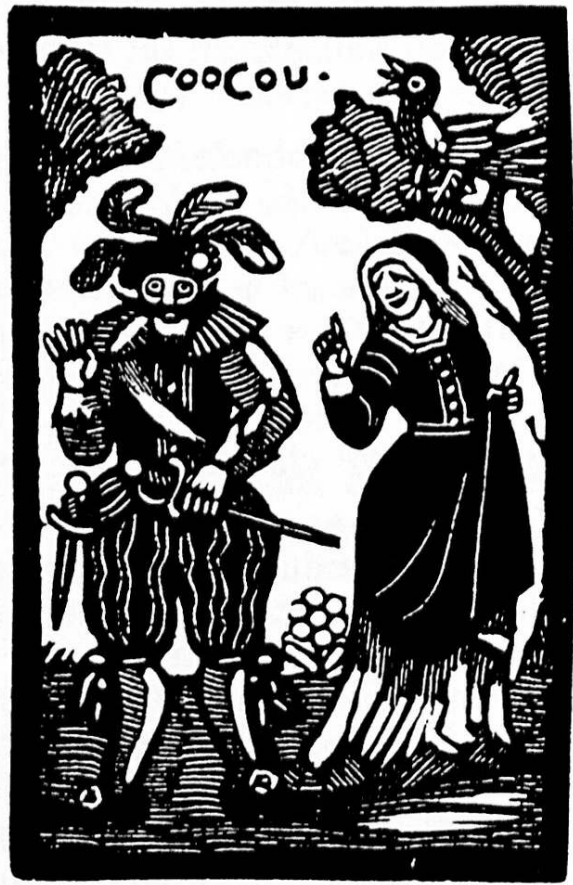

\section{NOTES}

1. Marie-Claude Hubert, Le théâtre, Armand Colin, « Cursus », Paris, 1988, pp. 40-41.

2. Jean-Claude Guy, Parole des anciens, Apophtegmes des pères du désert, Point Seuil, 1976, p. 17.

3. Montaigne, Essais, III, IX, « De la vanité ».

4. Pierre Voltz, La Comédie, Armand Colin, collection «U », 1964, p. 26. 


\section{AUTEUR}

\section{THIERRY MIGUET}

Université de Franche-Comté 